\title{
TERMINAL VALUE PROBLEM FOR SINGULAR ORDINARY DIFFERENTIAL EQUATIONS: THEORETICAL ANALYSIS AND NUMERICAL SIMULATIONS OF GROUND STATES
}

\author{
ALEX P. PALAMIDES AND THEODOROS G. YANNOPOULOS
}

Received 18 October 2005; Revised 26 July 2006; Accepted 13 August 2006

A singular boundary value problem (BVP) for a second-order nonlinear differential equation is studied. This BVP is a model in hydrodynamics as well as in nonlinear field theory and especially in the study of the symmetric bubble-type solutions (shell-like theory). The obtained solutions (ground states) can describe the relationship between surface tension, the surface mass density, and the radius of the spherical interfaces between the fluid phases of the same substance. An interval of the parameter, in which there is a strictly increasing and positive solution defined on the half-line, with certain asymptotic behavior is derived. Some numerical results are given to illustrate and verify our results. Furthermore, a full investigation for all other types of solutions is exhibited. The approach is based on the continuum property (connectedness and compactness) of the solutions funnel (Knesser's theorem), combined with the corresponding vector field's ones.

Copyright (c) 2006 A. P. Palamides and T. G. Yannopoulos. This is an open access article distributed under the Creative Commons Attribution License, which permits unrestricted use, distribution, and reproduction in any medium, provided the original work is properly cited.

\section{Introduction}

In order to study the behavior of nonhomogeneous fluids, Dell'Isola et al. [6] added an additional term to the volume-free energy $E_{0}(\rho)$ and hence the total energy of the fluid becomes

$$
E\left(\rho,|\nabla \rho|^{2}\right)=E_{0}(\rho)+\frac{\gamma}{2}|\nabla \rho|^{2}, \quad \gamma>0
$$

Then, under isothermal process, the D'Alembert-Lagrange principle can be applied (taking into account the conservation of mass) on the functional

$$
J(\rho, \overline{\boldsymbol{v}})=\int_{t_{1}}^{t_{1}} \int_{\Omega}\left(\rho \frac{|\overline{\boldsymbol{v}}|^{2}}{2}-E\left(\rho,|\nabla \rho|^{2}\right)\right) d \omega d t
$$

Hindawi Publishing Corporation

Boundary Value Problems

Volume 2006, Article ID 28719, Pages 1-28

DOI 10.1155/BVP/2006/28719 
to get the differential system

$$
\rho_{t}+\operatorname{div}(\rho \overline{\boldsymbol{v}})=0, \quad \frac{d \overline{\boldsymbol{v}}}{d t}+\nabla(\mu(\rho)-\gamma \Delta \rho)=0,
$$

where $\mu(\rho)=d E_{0}(\rho) / d \rho$ is the so called chemical potential of the fluid. When there is no motion of the fluid, this system is reduced to the equation

$$
\gamma \Delta \rho=\mu(\rho)-\mu_{0}
$$

where $\mu_{0}$ is a constant.

The differential equation (1.4) can be regarded as a model for microscopical spherical bubbles in a nonhomogeneous fluid. Because of the symmetry, we are interested in a solution depending only on the radial variable $\rho$. In that case [6] (see also [12]), (1.4) can be written as

$$
\left(r^{n-1} \rho^{\prime}(r)\right)^{\prime}=\frac{r^{n-1}}{\gamma} \mu(\rho)-\mu_{0}
$$

where $n=2,3, \ldots$, and it is known as the density profile equation. We must add boundary conditions on (1.5):

(i) because of the spherical symmetry, the derivative of $\rho$ must vanish at the origin

$$
\rho^{\prime}(0)=0
$$

(ii) since the bubble is surrounded by a liquid with density $\rho_{l}$, we must also have

$$
\lim _{r \rightarrow+\infty} \rho(r)=\rho_{l}>0
$$

We are interested in a strictly increasing solution $\rho=\rho(r)$ of the boundary value problem (1.5)-(1.7) with $0<\rho(r)<\rho_{l}$, a function describing an increasing mass density profile.

In the simple case under consideration, the chemical potential $\mu(\rho)$ is a third-degree polynomial on $\rho$ with three distinct positive roots $\rho_{1}<\rho_{2}<\rho_{3}=\rho_{l}$, that is, $\mu=\mu(\rho)=$ $4 \alpha\left(\rho-\rho_{1}\right)\left(\rho-\rho_{2}\right)\left(\rho-\rho_{3}\right)$. For $\lambda=\sqrt{\alpha / \gamma}\left(\rho_{2}-\rho_{1}\right)$ and $\xi=\left(\rho_{3}-\rho_{2}\right) /\left(\rho_{2}-\rho_{1}\right)$, the boundary value problem (1.5)-(1.7) can be written (without loss of generality) as

$$
\begin{gathered}
\frac{1}{r^{n-1}}\left(r^{n-1} \rho^{\prime}(r)\right)^{\prime}=4 \lambda^{2}(\rho+1) \rho(\rho-\xi):=f(\rho), \quad 0<r<+\infty, \\
\lim _{r \rightarrow 0+} r^{n-1} \rho^{\prime}(r)=0, \quad \lim _{r \rightarrow+\infty} \rho(r)=\xi .
\end{gathered}
$$

The solutions of this ordinary differential equation determine the mass density profile. Furthermore, BVPs of type (1.8) have also been used as models in the nonlinear field theory (see $[2,7]$ and the references therein). However the study of BVP (1.8) is not an easy subject (see [6, page 546]), but we endeavour to formulate a rigorous mathematical approach. Berestycki et al. [3] studied a generalized Emden equation and explained the physical significance of its solutions. In a recent paper [4], Bonheure et al. obtained some 
results on existence and multiplicity of the singular BVP

$$
\begin{gathered}
u^{\prime \prime}+k \frac{u^{\prime}}{t}=c(t) g(u), \\
u^{\prime}(0)=0, \quad u(M)=0,
\end{gathered}
$$

where $c(t)$ is bounded on $(0,+\infty)$ and $M \leq \infty$, combining shooting argument with variational methods.

For strongly singular higher-order linear differential equations together with twopoint conjugate and right-focal boundary conditions, Agarwal and Kiguradze [1] provided easily verifiable best possible conditions which guarantee the existence of a unique solution.

Using in this paper a quite different approach, we are going to prove, the existence of an increasing solution of (1.8) with a unique zero, at least for every $\xi \in\left(0, \xi_{M}\right)$, where the exact value of $\xi_{M}$ remains an open problem. Our estimation indicates that $\xi_{M} \simeq 0.83428$. As many previous studies pointed out, the existence of such a solution is a very important and meaningful case, in the above theories (bubble density, radius, surface tension, etc., are depending on it).

\section{Preliminaries: general theory}

Let us consider the following boundary value problem:

$$
\begin{gathered}
\frac{1}{p(r)}\left(p(r) \rho^{\prime}(r)\right)^{\prime}=f\left(r, \rho(r), p(r) \rho^{\prime}(r)\right), \\
\rho(0)=\rho_{0} \in(-1,0), \\
\lim _{r \rightarrow+\infty} \rho(r)=\xi,
\end{gathered}
$$

where $f: \Omega:=[0,+\infty) \times \mathbb{R}^{2} \rightarrow \mathbb{R}$ is continuous with three distinct zeros $-1,0$, and $\xi \in$ $(0,1)$, that is,

$$
f(t,-1, v)=f(t, 0, v)=f(t, \xi, v)=0 \quad \forall t \in(0,+\infty), v \in \mathbb{R},
$$

and further for all $t \in(0,+\infty)$ and $v \in \mathbb{R}$,

$$
f(t, u, v) \geq 0, \quad u \in(-1,0) \cup(\xi,+\infty), \quad f(t, u, v) \leq 0, \quad u \in(-\infty,-1] \cup(0, \xi) .
$$

Let us notice from the beginning that the constant functions

$$
\rho(r)=-1, \quad \rho(r)=0, \quad \rho(r)=\xi, \quad r \geq 0,
$$

are solutions of the equation in $(2.1)$ (with initial values $\rho(0)=-1, \rho(0)=0$, and $\rho(0)=$ $\xi$, resp.) and we will assume throughout of this section that they are unique.

Let us also suppose that $p \in C^{1}((0,+\infty),(0,+\infty))$ with $\lim _{t \rightarrow 0+} p(t)=0$ and

$$
\int_{0}^{t} p(r) d r<\infty, \quad \int_{0}^{t} \frac{1}{p(s)}\left\{\int_{0}^{s} p(x) d x\right\} d s<\infty \quad \text { for any } t>0 .
$$


4 A terminal BVP

Consider now the corresponding initial value problem

$$
\begin{aligned}
& \frac{1}{p(r)}\left(p(r) \rho^{\prime}(r)\right)^{\prime}-f\left(r, \rho(r), p(r) \rho^{\prime}(r)\right)=0, \\
& \rho(0)=\rho_{0} \in(-1,0), \quad \lim _{r \rightarrow 0+} p(r) \rho^{\prime}(r)=0,
\end{aligned}
$$

and prove the next existence results.

Proposition 2.1. Assume that the assumption (2.5) and the sign property on $f$ are fulfilled and further that there is a constant $M>0$ such that

$$
|f(t, u, v)| \leq M, \quad t \geq 0, u, v \in \mathbb{R} .
$$

Then the IVP (2.6) admits a global solution.

Proof. Let $\rho$ be a solution of (2.6). Then $\rho \in \mathscr{L}(P)$, the family of all solutions emanating from $P=\left(\rho_{0}, 0\right)$, implies

$$
\rho(t)=(S \rho)(t)
$$

where

$$
(S \rho)(t):=\rho_{0}+\int_{0}^{t} \frac{1}{p(s)} \int_{0}^{s} p(r) f\left(r, \rho(r), p(r) \rho^{\prime}(r)\right) d r d s
$$

For any (fixed) positive $T$, we may define the Banach space

$$
K^{1}[0, T]=\left\{u \in C[0, T], p u^{\prime} \in C[0, T]\right\}
$$

with norm

$$
\|u\|_{1}=\max \left\{\|u\|,\left\|p u^{\prime}\right\|\right\}
$$

where $\|u\|$ denotes the usual sup-norm of $u$ on $[0, T]$. On the other hand, in order to prove that the operator

$$
S: K^{1}[0, T] \longrightarrow K^{1}[0, T]
$$

is compact, we note that if $\rho_{0}$ takes values in a bounded set, there exist positives $K_{0}$ and $K_{1}$ such that

$$
\begin{aligned}
& |(S \rho)(t)| \leq\left|\rho_{0}\right|+M \int_{0}^{t} \frac{1}{p(s)} \int_{0}^{s} p(r) d r d s \leq K_{0}, \\
& \left|p(t)(S \rho)^{\prime}(t)\right| \leq M \int_{0}^{t} p(r) d r \leq K_{1}, \quad 0 \leq t \leq T .
\end{aligned}
$$

Then,

$$
\|S \rho\|_{1} \leq K=\max \left\{K_{0}, K_{1}\right\}
$$


Furthermore, $\{S \rho\}$ is an equicontinuous family since

$$
\begin{array}{r}
\left|(S \rho)(t)-(S \rho)\left(t^{\prime}\right)\right|=\left|\int_{t^{\prime}}^{t} \frac{1}{p(s)} \int_{0}^{s} p(r) f\left(r, \rho(r), p(r) \rho^{\prime}(r)\right) d r d s\right| \\
\leq M\left|\phi(t)-\phi\left(t^{\prime}\right)\right|, \\
\begin{aligned}
\left|p(t)(S \rho)^{\prime}(t)-p(t)(S \rho)^{\prime}\left(t^{\prime}\right)\right|<\left|\int_{t^{\prime}}^{t} p(r) f\left(r, \rho(r), p(r) \rho^{\prime}(r)\right) d r\right| \\
\leq M\left|\phi^{*}(t)-\phi^{*}\left(t^{\prime}\right)\right|, \quad 0 \leq t, t^{\prime} \leq T,
\end{aligned}
\end{array}
$$

and the mappings

$$
\phi(t)=\int_{0}^{t} \frac{1}{p(s)} \int_{0}^{s} p(r) d r d s, \quad \phi^{*}(t)=\int_{0}^{t} p(r) d r
$$

are absolutely continuous. Finally, by an application of the standard Schauder fixed-point theorem, we get a solution $\rho=\rho(r)$ defined over the entire interval $[0, T]$.

We consider now the segment

$$
E:=\left\{\left(\rho, p \rho^{\prime}\right): \rho=\rho_{0} \in(-1,0), p \rho^{\prime}=0\right\} .
$$

Theorem 2.2. Assume that the assumption (2.5) and the sign property on $f$ are fulfilled. Then (2.6) has a local solution $\rho \in \mathscr{X}(P), P \in E$.

Proof. Let $B:=\left\{(t, u, v): t \geq 0, \max \left\{\left\|u-\rho_{0}\right\|,\|v\|\right\}<1\right\}$. We associate to any $P \in[0, T] \times$ $\mathbb{R}^{2}$, the closest point $Q$ in $B$. This is obviously a continuous mapping. Defining the modification $g:[0, T] \times \mathbb{R}^{2} \rightarrow \mathbb{R}$ by $g(P)=f(Q)$, we see that $g$ is continuous, bounded, and $g=f$ on $B$. By the previous proposition, there is a solution $\rho \in \mathscr{X}(P)$ that solves the problem

$$
\begin{gathered}
\frac{1}{p(t)}\left(p(t) \rho^{\prime}(t)\right)^{\prime}=g\left(t, \rho(t), p(t) \rho^{\prime}(t)\right), \\
\rho(0)=\rho_{0}, \quad \lim _{r \rightarrow 0+} p(r) \rho^{\prime}(r)=0
\end{gathered}
$$

on $[0, T]$. Let

$$
\beta:=\sup \left\{s \in[0, T]:\left(t, \rho(t), p(t) \rho^{\prime}(t)\right) \in B \text { for } 0 \leq t \leq s\right\} .
$$

Evidently, $0<\beta \leq T$. On the other hand, since $g=f$ on $B$, we have

$$
\frac{1}{p(t)}\left(p(t) \rho^{\prime}(t)\right)^{\prime}=f\left(t, \rho(t), p(t) \rho^{\prime}(t)\right), \quad 0 \leq t \leq \beta,
$$

consequently, $\rho$ is a local solution of (2.6).

Taking into account the classical theorem of the extendability of solutions, we impose one more condition on the desired solution

$$
\lim _{r \rightarrow+\infty} p(r) \rho^{\prime}(r)=0 .
$$




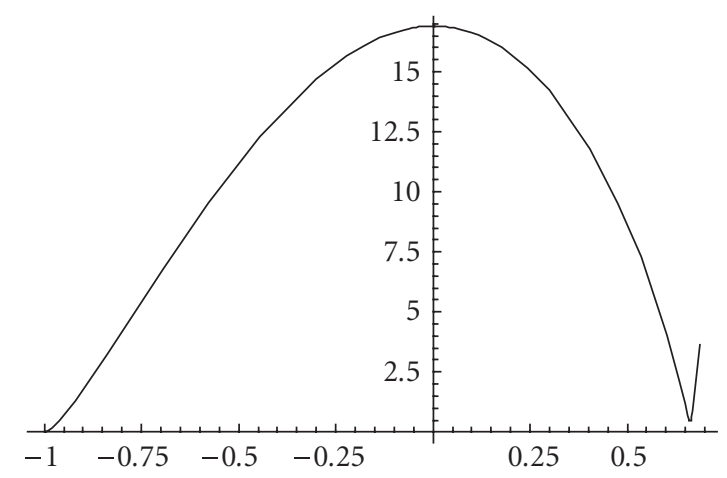

Figure 2.1. $\left(\xi \simeq 0.6616, \rho_{0} \simeq-0.999112\right)$.

Actually we seek for a strictly increasing solution of the differential equation in (2.1), which has (exactly) one zero and satisfies the asymptotic relationship $\lim _{r \rightarrow+\infty} \rho(r)=\xi$.

We notice now that a vector field can be defined on the phase plane, with crucial properties for our study. More precisely, noticing (2.3) and considering the $\left(\rho, p \rho^{\prime}\right)$ phase semiplane $\left(p \rho^{\prime} \geq 0\right)$, we easily check that

$$
\begin{array}{ll}
\left(p \rho^{\prime}\right)^{\prime}<0 & \text { for } \rho \in(-\infty,-1) \cup(0, \xi), \\
\left(p \rho^{\prime}\right)^{\prime}>0 & \text { for } \rho \in(-1,0) \cup(\xi,+\infty) .
\end{array}
$$

Thus, it is obvious that any solution of (2.6) with $\rho_{0} \geq \xi$ does not satisfy the demand $\lim _{r \rightarrow+\infty} \rho(r)=\xi$, since it is an increasing function. Similarly, whenever $\rho_{0} \leq-1$, the correspondingly solution $\rho=\rho(r), r \geq 0$, is not an increasing map. Consequently, the condition $\rho_{0} \in(-1,0)$ is necessary in order to obtain a solution with the desired properties and this is the reason for the restriction of the parameter $\rho_{0} \in(-1,0)$ in (2.6). Finally, any trajectory $\left(\rho(r), p(r) \rho^{\prime}(r)\right), r \geq 0$, emanating from the segment $E$, "moves" in a natural way (initially, when $\rho(r)<0$ ) toward the positive $p \rho^{\prime}$-semiaxis and then (when $\rho(r) \geq 0$ ) toward the positive $\rho$-semiaxis (see Figures 2.1-2.4). As a result, assuming a certain growth rate on $f$, we can control the vector field in such a way that it assures the existence of a trajectory satisfying the given properties and the boundary conditions

$$
\lim _{r \rightarrow+\infty} \rho(r)=\xi, \quad \lim _{r \rightarrow+\infty} p(r) \rho^{\prime}(r)=0 .
$$

These properties, will be referred to in the rest of this paper as "the nature of the vector field." Therefore, a combination of properties of the associated vector field with the Kneser's property of the cross sections of the solutions' funnel is the main tool that we will employ in our study. It is obvious therefore, that the technique presented here is different from those employed in the previous papers $[6,12]$, but closely related, at the same time, to the methods of $[9,11]$ or $[10]$.

For the convenience of the reader and to make the paper self-contained, we summarize here the basic notions used in the sequel. First, we refer to the well-known Kneser's theorem (see, e.g., the Copel's text book [5]). 


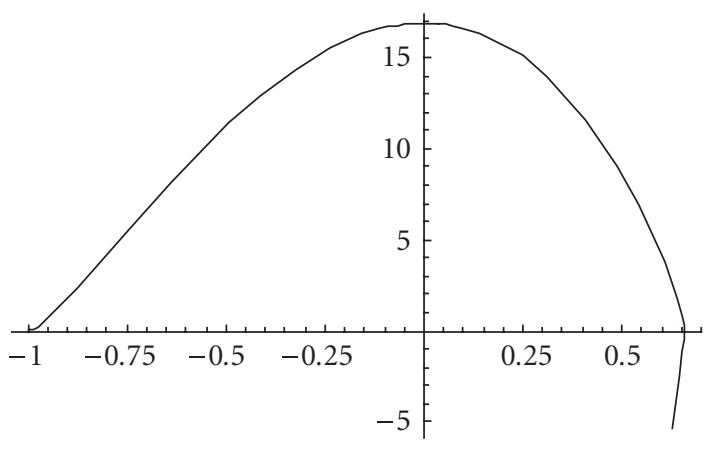

Figure 2.2. $\left(\xi \simeq 0.6617, \rho_{0} \simeq-0.999112\right)$.

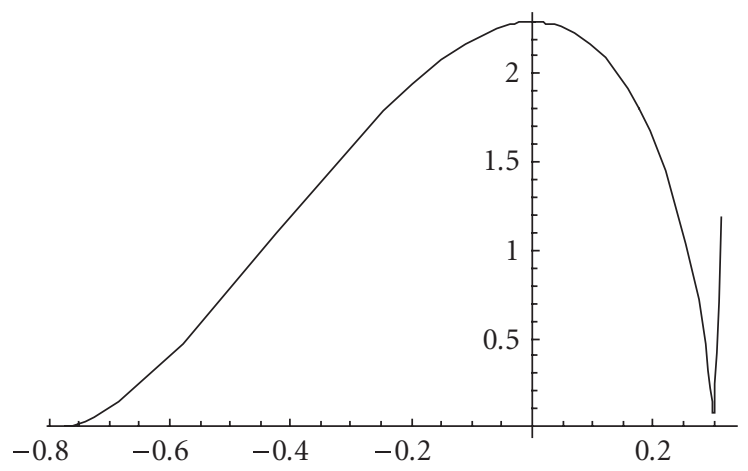

Figure 2.3. $\left(\rho_{0} \simeq-0.77075, \xi \simeq 0.3\right)$.

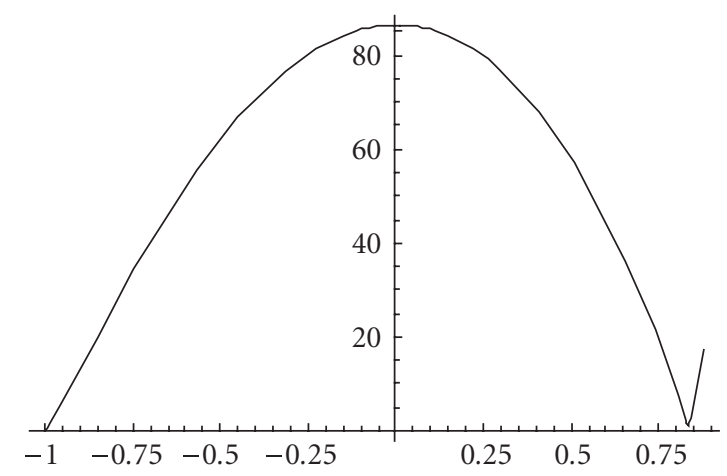

Figure 2.4. $\left(\rho_{0} \simeq-0.9999999932, \xi \simeq 0.83428\right)$.

Theorem 2.3. Consider the system

$$
y^{\prime}=f(x, y), \quad(x, y) \in[\alpha, \beta] \times \mathbb{R}^{n},
$$


with $f$ continuous and let $\hat{E}_{0}$ be a continuum (i.e., compact and connected) subset of $\mathbb{R}^{n}$ and let $\mathscr{X}\left(\hat{E}_{0}\right)$ be the family of all solutions of 2.24 emanating from $\hat{E}_{0}$. If any solution $y \in \mathscr{X}\left(\widehat{E}_{0}\right)$ is defined on the interval $[\alpha, \tau]$, then the cross section

$$
\mathscr{L}\left(\tau ; \hat{E}_{0}\right)=\left\{y(\tau): y \in \mathscr{L}\left(\widehat{E}_{0}\right)\right\}
$$

is a continuum in $\mathbb{R}^{n}$.

Reminding that a set-valued mapping $\mathscr{G}$, which maps a topological space $X$ into compact subsets of another one $Y$, is called upper semicontinuous (usc) at the point $x_{0}$ if and only if for any open subset $V$ in $Y$ with $\mathscr{G}\left(x_{0}\right) \subseteq V$ there exists a neighborhood $U$ of $x_{0}$ such that $\mathscr{G}(x) \subseteq V$ for every $x \in U$, we recall the next two lemmas, which were proved (without any assumption of uniqueness of solutions) in [9].

Lemma 2.4. Let $X$ and $Y$ be metric spaces and let $\varphi: X \rightarrow 2^{Y}$ be a usc mapping. If $A$ is a continuum subset of $X$ such that, for every $x \in A$, the set $\mathscr{G}(x)$ is a continuum, then the image $\mathscr{G}(A):=\cup\{\mathscr{G}(x): x \in A\}$ is also a continuum subset of $Y$.

We consider the set

$$
\omega:=\left\{\left(\rho, p \rho^{\prime}\right):-1 \leq \rho<\xi, p \rho^{\prime} \geq 0\right\}
$$

any point $P_{0}:=\left(\rho_{0}, \rho_{0}^{\prime}\right) \in E \subseteq \partial \omega$ and the family $\mathscr{X}\left(P_{0}\right)$ of all noncontinuable solutions of the initial value problem (2.6). By the continuity of the nonlinearity and the nature of the vector field (sign of $f$ ), we have two possible cases.

(i) Considering a solution $\rho \in \mathscr{X}\left(P_{0}\right)$, there exists $r_{1} \geq 0$ (depending on $\rho$ ) such that

$$
p\left(r_{1}\right) \rho^{\prime}\left(r_{1}\right)=0, \quad \rho\left(r_{1}\right)<\xi, \quad \text { or } \quad p\left(r_{1}\right) \rho^{\prime}\left(r_{1}\right)>0, \quad \rho\left(r_{1}\right)=\xi,
$$

and furthermore the restriction $\rho \mid\left[0, r_{1}\right]$ is an increasing function. Consequently in this case, we can define a map $\mathscr{K}: E \rightarrow 2^{\partial \omega}$ by

$$
\mathscr{H}\left(P_{0}\right):=\left\{\left(\rho\left(r_{1}\right), p\left(r_{1}\right) \rho^{\prime}\left(r_{1}\right)\right) \in \partial \omega: \rho \in \mathscr{X}\left(P_{0}\right)\right\}
$$

(ii) In the case where $\mathscr{K}(E)=\cup\left\{\mathscr{K}\left(P_{0}\right): P_{0} \in E\right\} \neq \varnothing$ and there a point $P_{0} \in E$ such that $\operatorname{Dom}(\rho)=[0,+\infty)$ and

$$
\lim _{r \rightarrow+\infty} p(r) \rho^{\prime}(r)=0, \quad \lim _{r \rightarrow+\infty} \rho(r)=\xi
$$

for some $\rho \in \mathscr{X}\left(P_{0}\right)$, we will say that $P_{0}$ is a singular point of the above map $\mathscr{K}$. This is exactly the case, the existence of which we must investigate.

Lemma 2.5 [9]. The above mapping $\mathscr{K}$ is upper semicontinuous (usc) at any nonsingular point $P_{0}:=\left(\rho_{0}, \rho_{0}^{\prime}\right) \in E$ and the set $\mathscr{K}\left(P_{0}\right)$ is a continuum. Moreover, the image $\mathscr{K}(B)$ of any continuum $B$ is also a connected and compact set.

We also need another lemma from the classical topology. 
Lemma 2.6 (see [8, Chapter V, Paragraph 47, point III, Theorem 2]). If $A$ is an arbitrary proper subset of a continuum $B$ and $S$ a connected component of $A$, then

$$
\bar{S} \cap \overline{(B \backslash A)} \neq \varnothing,
$$

that is,

$$
\bar{S} \cap \partial A \neq \varnothing .
$$

Let $A$ be a subset of $\omega$. We set

$$
\mathscr{L}(A):=\cup\{\mathscr{X}(P): P \in A\}
$$

and recall that $\mathscr{L}\left(r^{*} ; A\right):=\left\{\left(\rho\left(r^{*}\right), p\left(r^{*}\right) \rho^{\prime}\left(r^{*}\right)\right): \rho \in \mathscr{X}(A)\right\}$ represents the cross-section of all solutions $\rho \in \mathscr{X}(A)$ at the point $r=r^{*}$. For the domain $\omega$, let $\mathscr{K}$ denote the above mapping, which is defining with respect to the set $\omega$. Then the following lemma holds.

Lemma 2.7. If the subset $E_{0} \subset E$ is a continuum such that

$$
\mathscr{K}\left(E_{0}\right) \cap E_{\xi}^{*} \neq \varnothing, \quad \mathscr{K}\left(E_{0}\right) \cap E^{*} \neq \varnothing
$$

and contains exactly one singular point $P_{0}:=\left(\rho_{0}, p \rho_{0}^{\prime}\right)$ of the map $\mathcal{K}$, then both the sets $\mathscr{K}\left(E_{0}\right) \cap E_{\xi}^{*}$ and $\mathscr{K}\left(E_{0}\right) \cap E^{*}$ are bounded and connected subsets of $\partial \omega$, where

$$
E_{\xi}^{*}=\left\{\left(\rho, p \rho^{\prime}\right) \in \partial \omega: \rho=\xi\right\}, \quad E^{*}:=\left\{\left(\rho, p \rho^{\prime}\right) \in \partial \omega: p \rho^{\prime}=0\right\} .
$$

Proof. By the continuation of solutions and the singularity of $\mathscr{Y}$ at the point $P_{0}$, the set $\mathscr{K}\left(P_{0}\right)=\varnothing$. Taking into account the nature of the vector field and the definition of the singularity of the map $\mathscr{K}$, this means that

$$
\lim _{r \rightarrow+\infty} p(r) \rho^{\prime}(r)=0, \quad \lim _{r \rightarrow+\infty} \rho(r)=\xi .
$$

Since $P_{0}$ separates $E_{0}$ into two bounded connected sets, the result follows by the continuity of $\mathscr{K}$ and the uniqueness of the solution $\rho(r)=\xi$.

Proposition 2.8. Let $P_{0}=\left(\rho_{0}, p \rho_{0}^{\prime}\right) \in E_{0}$ be a singular point of the consequent map $\mathscr{K}$, where $E_{0} \subset E$ is a continuum. Then, every connected component $S$ of the (assuming nonempty) set $S=E^{*} \cap \mathcal{K}\left(E_{0}\right)$ approaches the boundary $E_{\xi}^{*}$ of $\partial \omega$ in the sense that $\bar{S} \cap \partial E_{\xi}^{*} \neq \varnothing$.

Proof. By Lemma 2.7, the set $B=\left(E^{*} \cup E_{\xi}^{*}\right) \cap\left(\mathscr{K}\left(E_{0}\right) \cup\{(\xi, 0)\}\right)$ is a continuum. The set $A=E^{*} \cap \mathscr{K}\left(E_{0}\right)$ is a connected subset of $B$. Then the same set $S=E^{*} \cap \mathscr{K}\left(E_{0}\right)$ is a connected subset of $A$. Therefore, an ample use of Lemma 2.6 gives $\bar{S} \cap \partial E_{\xi}^{*} \neq \varnothing$.

Now we give a theorem which summarizes the main results, concerning the existence of a solution of the boundary value problem, under consideration.

Theorem 2.9. Let also $E_{0}$ be a continuum in E such that

$$
\mathscr{K}\left(E_{0}\right) \cap E^{*} \neq \varnothing, \quad \mathscr{K}\left(E_{0}\right) \cap E_{\xi}^{*} \neq \varnothing .
$$

Then the boundary value problem (2.1)-(2.21) admits a strictly increasing solution. 
Proof. The result follows by Proposition 2.8.

Remark 2.10. In view of the above procedure and since by assumption $\lim _{t \rightarrow 0+} p(t)=0$, it is clear that the second initial condition $\lim _{r \rightarrow 0+} p(r) \rho^{\prime}(r)=0$ in (2.6) can be relax to any one of the form $\lim _{r \rightarrow 0+} p^{*}(r) \rho^{\prime}(r)=0$, where the new function $p^{*}(r)>0, r>0$, satisfies also the restriction (2.5) and

$$
\lim _{r \rightarrow 0+} p^{*}(r) \rho^{\prime}(r)=0 \Longrightarrow \lim _{r \rightarrow 0+} p(r) \rho^{\prime}(r)=0 .
$$

In particular, if $\lim _{t \rightarrow 0+} p^{*}(t)=l>0$, for example whenever $p^{*}(t)=1$ is the constant map, then (2.5) are fulfilled automatically, that is, the boundary conditions in (2.6) can read as

$$
\rho(0)=\rho_{0} \in(-1,0), \quad \lim _{r \rightarrow 0+} \rho^{\prime}(r)=0 .
$$

\section{Main results}

Consider the following singular boundary value problem:

$$
\begin{gathered}
\frac{1}{r^{n-1}}\left(r^{n-1} \rho^{\prime}(r)\right)^{\prime}=4 \lambda^{2}(\rho+1) \rho(\rho-\xi):=f(\rho), \\
\lim _{r \rightarrow 0+} r^{n-1} \rho^{\prime}(r)=0, \quad \lim _{r \rightarrow+\infty} \rho(r)=\xi,
\end{gathered}
$$

modeling the density profile problem.

Since $\lim _{\rho \rightarrow 0}(f(\rho) / \rho)=-4 \lambda^{2} \xi$ for every $\varepsilon \in(0, \xi)$, there exists an $\eta \in(0,1)$ such that

$$
\begin{gathered}
-4 \lambda^{2}(\xi+\varepsilon) \rho \leq f(\rho) \leq 4 \lambda^{2}(-\xi+\varepsilon) \rho \leq 0, \quad 0 \leq \rho \leq \eta, \\
0 \leq 4 \lambda^{2}(-\xi+\varepsilon) \rho \leq f(\rho) \leq-4 \lambda^{2}(\xi+\varepsilon) \rho, \quad-\eta \leq \rho \leq 0 .
\end{gathered}
$$

Consider the corresponding initial value problem

$$
\begin{gathered}
\frac{1}{r^{n-1}}\left(r^{n-1} \rho^{\prime}(r)\right)^{\prime}=4 \lambda^{2}(\rho+1) \rho(\rho-\xi):=f(\rho), \\
\rho(0)=-\eta, \quad \lim _{r \rightarrow 0+} r^{n-1} \rho^{\prime}(r)=0 .
\end{gathered}
$$

In view of Theorem 2.2 and Remark 2.10, this singular IVP has a local solution. By the nature of the vector field (sign of the nonlinearity), any solution $\rho=\rho(r)$ of (3.3) as well as its derivative $r^{n-1} \rho^{\prime}(r)$ are strictly increasing functions in a (right) neighborhood of $r=0$, precisely as far as $\rho(r) \leq 0$. With respect to the existence of $\rho=\rho(r)$, we notice that the point $r=0$ is a regular singularity for the equation in (3.3) (see, e.g., [14] or [13]). Precisely, this initial value problem has a unique solution, which is a holomorphic function at the point $r=0$, that is,

$$
\rho(r)=-\eta+\sum_{k=1}^{+\infty} \rho_{2 k}(-\eta) r^{2 k}, \quad 0 \leq r \leq \delta
$$


where the coefficients $\rho_{2 k}=\rho_{2 k}(-\eta)$ are given by a recurrence formulae, for example,

$$
\rho_{2}(-\eta)=\left(2 \lambda^{2} / n\right)(-\eta)(-\eta+1)(-\eta-\xi) .
$$

Remark 3.1. Although the initial condition $\lim _{r \rightarrow 0+} r^{n-1} \rho^{\prime}(r)=0$ in (3.3) seems to be weaker than the natural boundary condition $\lim _{r \rightarrow 0+} \rho^{\prime}(r)=0$ (see (1.6)), in the present situation the later follows. Indeed, since

$$
\lim _{r \rightarrow 0+} \frac{\left(r^{n-1} \rho^{\prime}(r)\right)^{\prime}}{r^{n-1}}=4 \lambda^{2}(-\eta+1)(-\eta)(-\eta-\xi)=\theta_{0}>0
$$

for any small enough $\varepsilon>0$,

$$
0 \leq\left(r^{n-1} \rho^{\prime}(r)\right)^{\prime} \leq\left(\theta_{0}+1\right) r^{n-1}, \quad 0 \leq r \leq \varepsilon .
$$

Hence, an integration on the interval $[0, \varepsilon]$ yields

$$
\rho^{\prime}(\varepsilon) \leq \frac{\left(\theta_{0}+1\right)}{n} \varepsilon,
$$

that is,

$$
\lim _{r \rightarrow 0+} \rho^{\prime}(r)=0 .
$$

Lemma 3.2. For any (small) $y_{0}>0$, there exists an $\eta_{0} \in[0, \eta)$ and $r_{1}>0$ such that the solution $\rho=\rho(r)$ of (3.3), (with $\eta$ replaced by $\eta_{0}$ ) satisfies

$$
-\eta_{0} \leq \rho(r)<0, \quad \rho\left(r_{1}\right)=0, \quad 0 \leq r^{n-1} \rho^{\prime}(r) \leq y_{0}, \quad 0 \leq r<r_{1} .
$$

Proof. We assume that there is not any $r_{1}>0$ for which the first of $(3.10)$ is fulfilled. Then, let us suppose that

$$
\rho(r) \leq 0, \quad r \geq 0 .
$$

In view of (3.1)-(3.3) and recalling the nature of the vector field, we have

$$
\left(r^{n-1} \rho^{\prime}(r)\right)^{\prime} \geq 4 \lambda^{2}(-\xi+\varepsilon) \rho(r) r^{n-1}, \quad 0 \leq r<+\infty .
$$

Consequently, $\left(r^{n-1} \rho^{\prime}(r)\right)^{\prime} \geq 0,0 \leq r<+\infty$, so $r^{n-1} \rho^{\prime}(r)>0,0 \leq r<+\infty$ and further this means that the solution $\rho=\rho(r), 0 \leq r<+\infty$ is an increasing map. Hence,

$$
\lim _{r \rightarrow+\infty} \rho(r)=l \leq 0,
$$

and this implies

$$
\lim _{r \rightarrow+\infty} \rho^{\prime}(r)=0 .
$$

Now given that

$$
\lim _{r \rightarrow 0+} r \rho^{\prime}(r)=0 \Rightarrow \lim _{r \rightarrow 0+} r^{n-1} \rho^{\prime}(r)=0,
$$


an integration of (3.12) on the interval $[0, r]$ yields

$$
r^{n-1} \rho^{\prime}(r) \geq-4 \lambda^{2}(\xi-\varepsilon) \int_{0}^{r} \rho(t) t^{n-1} d t, \quad 0 \leq r<+\infty .
$$

We notice first that for $l=0$ (by the L'Hospital's rule),

$$
\lim _{t \rightarrow+\infty} t^{n-1}[-\rho(t)]=\lim _{t \rightarrow+\infty} \frac{-\rho(t)}{1 / t^{n-1}}=\frac{1}{n-1} \lim _{t \rightarrow+\infty} t^{n} \rho^{\prime}(t)=+\infty,
$$

because the function $r^{n-1} \rho^{\prime}(r)$ is positive and increasing. Hence,

$$
\lim _{r \rightarrow+\infty} \int_{0}^{r}[-\rho(t)] t^{n-1} d t=+\infty .
$$

If $l<0$, then (3.18) is still true and further

$$
\lim _{r \rightarrow+\infty} \rho^{\prime}(t) \geq 4 \lambda^{2}(\xi-\varepsilon) \lim _{r \rightarrow+\infty} \frac{\int_{0}^{r}[-\rho(t)] t^{n-1} d t}{r^{n-1}}=-\frac{4 \lambda^{2}(\xi-\varepsilon)}{n-1} \lim _{r \rightarrow+\infty} r \rho(r)=+\infty,
$$

a contradiction to (3.14). Let us now assume that $l=0$. Then by (3.14), we have $\lim _{r \rightarrow+\infty} \rho^{\prime}(r)=0$ and then noticing (3.16),

$$
\begin{aligned}
0 & =\lim _{r \rightarrow+\infty} \rho^{\prime}(r) \geq-4 \lambda^{2}(-\xi+\varepsilon) \lim _{r \rightarrow+\infty} \frac{\int_{0}^{r}[-\rho(t)] t^{n-1} d t}{r^{n-1}} \\
& =-\frac{4 \lambda^{2}(-\xi+\varepsilon)}{n-1} \lim _{r \rightarrow+\infty} \frac{r^{n-1}[-\rho(r)]}{r^{n-2}} \\
& =-\frac{4 \lambda^{2}(-\xi+\varepsilon)}{n-1} \lim _{r \rightarrow+\infty} \frac{\rho(r)}{1 / r}=\frac{4 \lambda^{2}(\xi-\varepsilon)}{n-1} \lim _{r \rightarrow+\infty} r^{2} \rho^{\prime}(r) \geq 0,
\end{aligned}
$$

provided that the last limit $\lim _{r \rightarrow+\infty} r^{2} \rho^{\prime}(r)$ exists.

In order to demonstrate this assertion, we notice first that

$$
\lim _{r \rightarrow+\infty} r^{n-1} \rho^{\prime}(r)=m \leq+\infty
$$

because $\left(r^{n-1} \rho^{\prime}(r)\right)^{\prime} \geq 0,0 \leq r<+\infty$. Now since

$$
\lim _{r \rightarrow+\infty} r^{2} \rho^{\prime}(r)=\lim _{r \rightarrow+\infty} \frac{r^{n-1} \rho^{\prime}(r)}{r^{n-3}}
$$

we immediately get

$$
\lim _{r \rightarrow+\infty} r^{2} \rho^{\prime}(r)=\left\{\begin{array}{ll}
m & \text { if } n=3, \\
0 & \text { if } n>3,
\end{array} \quad m<+\infty .\right.
$$

So assume that $n>3$ and $m=+\infty$. Then

$$
\lim _{r \rightarrow+\infty} r^{2} \rho^{\prime}(r)=\lim _{r \rightarrow+\infty} \frac{\left(r^{n-1} \rho^{\prime}(r)\right)^{\prime}}{\left(r^{n-3}\right)^{\prime}}=\lim _{r \rightarrow+\infty} \frac{r^{n-1} 4 \lambda^{2}(\rho(r)-1) \rho(r)(\rho(r)-\xi)}{(n-3) r^{n-4}},
$$


given that the limit

$$
\lim _{r \rightarrow+\infty} r^{3}[-\rho(r)]=\lim _{r \rightarrow+\infty} \frac{[-\rho(r)]}{r^{-3}}
$$

exists. One more application of the L'Hospital's rule guarantee, that (3.25) exists if

$$
\lim _{r \rightarrow+\infty} r^{4} \rho^{\prime}(r)
$$

exists too. As above

$$
\lim _{r \rightarrow+\infty} r^{4} \rho^{\prime}(r)= \begin{cases}+\infty & \text { if } n=3,4, \\ m & \text { if } n=5, \quad m<+\infty . \\ 0 & \text { if } n>5,\end{cases}
$$

For $n>5$ and $m=+\infty$, we similarly get

$$
\lim _{r \rightarrow+\infty} r^{4} \rho^{\prime}(r)=\lim _{r \rightarrow+\infty} \frac{r^{n-1} 4 \lambda^{2}(\rho(r)-1) \rho(r)(\rho(r)-\xi)}{(n-5) r^{n-6}},
$$

given that

$$
\lim _{r \rightarrow+\infty} \frac{[-\rho(r)]}{r^{-5}}
$$

exists.

Continuing this procedure, we conclude that the $\operatorname{limit}_{\lim _{r \rightarrow+\infty}} r^{2} \rho^{\prime}(r)$ exists if at least one of

$$
\lim _{r \rightarrow+\infty} r^{n-1}[-\rho(r)] \quad \text { or } \quad \lim _{r \rightarrow+\infty} r^{n-1} \rho^{\prime}(r)
$$

exists. But this is true (see (3.17) or (3.21)).

This is a contradiction if $n \leq 3$, in view of (3.16). If $n>3$, we assert that there exists a sequence $\left\{r_{\nu}\right\}$ with $\lim r_{v}=+\infty$, such that $\lim r_{\nu}^{2} \rho^{\prime}\left(r_{\nu}\right)>0$ and this clearly contradicts the above equality $\lim _{r \rightarrow+\infty} r^{2} \rho^{\prime}(r)=0$. In order to demonstrate the last assertion, let us suppose that $\lim r_{\nu}^{2} \rho^{\prime}\left(r_{\nu}\right)=0$ for any such sequence. On the other hand, we know that $\lim _{r \rightarrow+\infty} r^{n-1} \rho^{\prime}(r)>0$ and so let

$$
k=\max \left\{m=2,3, \ldots, n-2: \exists r_{\nu} \longrightarrow \infty, \lim r_{\nu}^{m} \rho^{\prime}\left(r_{\nu}\right)=0\right\} .
$$

Then since $\lim _{r \rightarrow+\infty} r^{n-1} \rho^{\prime}(r)>0$, it is clear that $k \leq n-3$ and further by maximality of $k$, there is a subsequence of $\left\{r_{\nu}\right\}$, say itself such that

$$
\lim r_{\nu}^{k} \rho^{\prime}\left(r_{\nu}\right)=0, \quad \lim r_{\nu}^{k+2} \rho^{\prime}\left(r_{\nu}\right)>0 .
$$

Then again (3.16) implies

$$
r_{\nu}^{k} \rho^{\prime}\left(r_{\nu}\right) \geq-4 \lambda^{2}(\xi-\varepsilon) \frac{1}{r_{\nu}^{n-1-k}} \int_{0}^{r_{\nu}} \rho(t) t^{n-1} d t, \quad 0 \leq r_{\nu}<+\infty,
$$


14 A terminal BVP

and hence, given that

$$
\lim _{r \rightarrow+\infty} \frac{\int_{0}^{r}[-\rho(t)] t^{n-1} d t}{r^{n-1-k}}=\frac{1}{n-1-k} \lim _{r \rightarrow+\infty} \frac{r^{n-1}[-\rho(r)]}{r^{n-2-k}},
$$

it follows that

$$
\begin{aligned}
0 & =\lim r_{\nu}^{k} \rho^{\prime}\left(r_{\nu}\right) \geq 4 \lambda^{2}(\xi-\varepsilon) \lim \frac{\int_{0}^{r_{\nu}}[-\rho(t)] t^{n-1} d t}{r_{\nu}^{n-1-k}}=\frac{4 \lambda^{2}(\xi-\varepsilon)}{n-1-k} \lim \frac{r_{\nu}^{n-1}\left[-\rho\left(r_{\nu}\right)\right]}{r_{\nu}^{n-2-k}} \\
& =\frac{4 \lambda^{2}(-\xi+\varepsilon)}{n-1-k} \lim \frac{\rho^{\prime}\left(r_{\nu}\right)}{(-k-1) r_{\nu}^{-k-2}}=\frac{4 \lambda^{2}(\xi-\varepsilon)}{(n-1-k)(k+1)} \lim r_{\nu}^{k+2} \rho^{\prime}\left(r_{\nu}\right)>0,
\end{aligned}
$$

a contradiction.

Consequently for each $\eta_{0} \in[0, \eta]$, there is an $r_{\eta_{0}}>0$ such that

$$
-\eta_{0} \leq \rho(r) \leq 0, \quad 0 \leq r<r_{\eta_{0}}, \quad \rho\left(r_{\eta_{0}}\right)=0 .
$$

Consider now the set

$$
\omega_{0}=\left\{\left(\rho, r^{n-1} \rho^{\prime}\right):-\eta \leq \rho \leq 0, r^{n-1} \rho^{\prime} \geq 0\right\}
$$

and define a map $\mathcal{K}_{0}: E_{0}=[-\eta, 0] \times\{0\} \rightarrow 2^{\partial \omega_{0}}$ by the formula

$$
\mathscr{K}_{0}\left(-\eta_{0}, 0\right)=\left(\rho\left(r_{\eta_{0}}\right), r_{\eta_{0}}^{n-1} \rho^{\prime}\left(r_{\eta_{0}}\right)\right)
$$

Clearly the image $\mathscr{K}_{0}\left(E_{0}\right)$ is a continuum. Thus the point

$$
r_{\eta}=\max \left\{r_{\eta_{0}}: \eta_{0} \in[0, \eta]\right\}
$$

is finite and independent $\eta_{0}$.

On the other hand, by (3.2), we also have

$$
\left(r^{n-1} \rho^{\prime}(r)\right)^{\prime} \leq-4 \lambda^{2}(\xi+\varepsilon) \rho(r) r^{n-1}, \quad 0 \leq r<r_{\eta_{0}} \leq r_{\eta}
$$

and so

$$
\begin{aligned}
r_{\eta_{0}}^{n-1} \rho^{\prime}\left(r_{\eta_{0}}\right) & \leq-4 \lambda^{2}(\xi+\varepsilon) \int_{0}^{r_{\eta_{0}}} \rho(r) r^{n-1} d r \leq-4 \lambda^{2}(\xi+\varepsilon) \rho(0) \int_{0}^{r_{\eta_{0}}} r^{n-1} d r \\
& =-4 \lambda^{2}(\xi+\varepsilon) \frac{r_{\eta_{0}}^{n}}{n} \rho(0)=4 \lambda^{2}(\xi+\varepsilon) \frac{r_{\eta_{0}}^{n}}{n} \eta_{0} .
\end{aligned}
$$

Hence we get

$$
r_{\eta_{0}}^{n-1} \rho^{\prime}\left(r_{\eta_{0}}\right) \leq 4 \lambda^{2}(\xi+\varepsilon) \eta_{0} \frac{r_{\eta}^{n}}{n}
$$


that is, we may choose

$$
\eta_{0} \leq \min \left\{\eta, \frac{n}{(\xi+\varepsilon) 4 \lambda^{2} r_{\eta}^{n}} y_{0}\right\}
$$

and then clearly (3.10) is fulfilled.

Lemma 3.3. Consider any $\eta_{1} \leq \eta$, then there is a (small enough) $y_{0}^{*}$ such that for every positive $y_{0} \leq y_{0}^{*}$ the corresponding solution $\rho=\rho(r)$ with initial value $\rho\left(r_{1}\right)=0, r_{1}^{n-1} \rho^{\prime}\left(r_{1}\right)=y_{0}$ satisfies

$$
0 \leq \rho(r)<\eta_{1}, \quad y_{0} \geq r^{n-1} \rho^{\prime}(r)>0, \quad r_{1} \leq r<r_{2}, r_{2}^{n-1} \rho^{\prime}\left(r_{2}\right)=0
$$

for some $r_{2}>r_{1}$.

Proof. Let us suppose on the contrary that an arbitrary small point $y_{0}$ exists, with

$$
r^{n-1} \rho^{\prime}(r)>0, \quad r_{1} \leq r<+\infty .
$$

We will show there exists an $r_{2}>r_{1}$ such that

$$
\rho\left(r_{2}\right)=\eta_{1}
$$

Assume on the contrary that

$$
0 \leq \rho(r)<\eta_{1}, \quad r \geq r_{1}
$$

Since the function $r^{n-1} \rho^{\prime}(r), r \geq r_{1}$, is decreasing,

$$
\lim _{r \rightarrow+\infty} r^{n-1} \rho^{\prime}(r)=m \geq 0 .
$$

Hence

$$
\lim _{r \rightarrow+\infty} \rho^{\prime}(r)=0, \quad \text { and then } \lim _{r \rightarrow+\infty} \rho(r)=l \in\left(0, \eta_{1}\right) \text {. }
$$

Now in view of (3.2),

$$
\left(r^{n-1} \rho^{\prime}(r)\right)^{\prime} \leq 4 \lambda^{2}(-\xi+\varepsilon) \rho(r) r^{n-1}, \quad r_{1} \leq r<+\infty,
$$

and this yields the contradiction

$$
\begin{aligned}
\lim _{r \rightarrow+\infty} \rho^{\prime}(t) & \leq 4 \lambda^{2}(-\xi+\varepsilon) \lim _{r \rightarrow+\infty} \frac{\int_{r_{1}}^{r}[\rho(t)] t^{n-1} d t}{r^{n-1}}-\lim _{r \rightarrow+\infty} \frac{y_{0}}{r^{n-1}} \\
& =\frac{4 \lambda^{2}(-\xi+\varepsilon)}{n-1} \lim _{r \rightarrow+\infty} r \rho(r)=-\infty .
\end{aligned}
$$

Thus (3.46) holds. 
We fix a point $\hat{y}_{0}>0$ and we will prove first that the set

$$
\left\{\begin{array}{c}
r_{2}>r_{1}: \exists y_{0} \in\left(0, \hat{y}_{0}\right] \text { such that the corresponding solution with } \\
\rho\left(r_{1}\right)=0, r_{1}^{n-1} \rho^{\prime}\left(r_{1}\right)=y_{0} \text { satisfies }(3.45)-(3.46)
\end{array}\right\}
$$

is bounded, say by $r_{2}^{*}$. Assume on the contrary, that there exist sequences

$$
\left\{y_{0 k}\right\} \subset\left(0, \hat{y}_{0}\right], \quad\left\{r_{2, k}\right\} \text { with } \lim r_{2, k}=+\infty
$$

such that the corresponding solutions $\left\{\rho_{k}\right\}$ satisfy

$$
0 \leq \rho_{k}(r)<\eta_{1}, \quad y_{0 k} \geq r^{n-1} \rho_{k}^{\prime}(r)>0, \quad r_{1} \leq r<r_{2, k}, \quad \rho_{2 k}\left(r_{2, k}\right)=\eta_{1} .
$$

Then by (3.2) and (3.45), we get

$$
\left(r^{n-1} \rho_{k}^{\prime}(r)\right)^{\prime} \leq 4 \lambda^{2}(-\xi+\varepsilon) \rho_{k}(r) r^{n-1}, \quad r_{1} \leq r<r_{2, k} .
$$

Thus, an integration on the interval $\left[r_{1}, r_{2, k}\right]$ yields

$$
\begin{aligned}
r_{2, k}^{n-1} \rho_{k}^{\prime}\left(r_{2, k}\right) & \leq y_{0 k}+4 \lambda^{2}(-\xi+\varepsilon) \int_{r_{1}}^{r_{2, k}} \rho_{k}(t) t^{n-1} d t \\
& =y_{0 k}+4 \lambda^{2}(-\xi+\varepsilon)\left[\frac{r_{2, k}^{n}}{n} \rho_{k}\left(r_{2, k}\right)-\frac{r_{1}^{n}}{n} \rho_{k}\left(r_{1}\right)-\int_{r_{1}}^{r_{2, k}} \frac{t^{n}}{n} \rho_{k}^{\prime}(t) d t\right] \\
& \leq y_{0 k}+4 \lambda^{2}(-\xi+\varepsilon) \frac{r_{2, k}^{n}}{n} \eta_{1}-4 \lambda^{2}(-\xi+\varepsilon) y_{0 k} \frac{r_{2, k}^{2}-r_{1}^{2}}{2 n}, \quad r \in\left[r_{1}, r_{2, k}\right] .
\end{aligned}
$$

Hence we get

$$
\rho_{k}^{\prime}\left(r_{2, k}\right) \leq \frac{y_{0 k}}{r_{2, k}^{n-1}}\left[1-4 \lambda^{2}(-\xi+\varepsilon) \frac{r_{2, k}^{2}-r_{1}^{2}}{2 n}\right]+4 \lambda^{2}(-\xi+\varepsilon) \frac{r_{2, k}}{n} \eta_{1},
$$

and then for all large $k$, we conclude the contradiction $\rho_{k}^{\prime}\left(r_{2, k}\right)<0$.

We set now

$$
y_{0}^{*}=\min \left\{\hat{y}_{0}, \frac{4 \lambda^{2}(\xi-\varepsilon)\left(r_{1}^{n} / n\right) \eta_{1}}{1+4 \lambda^{2}(\xi-\varepsilon)\left(r_{2}^{* n}-r_{1}^{n}\right) / 2 n}\right\}
$$

and consider any

$$
y_{0} \in\left(0, y_{0}^{*}\right)
$$

such that (3.45)-(3.46) are fulfilled. Then again by (3.2), we get

$$
\left(r^{n-1} \rho^{\prime}(r)\right)^{\prime} \leq 4 \lambda^{2}(-\xi+\varepsilon) \rho(r) r^{n-1}, \quad r_{1} \leq r<r_{2} .
$$


Thus, noticing (3.58) and the definition of $r_{2}^{*}$, an integration on the interval $\left[r_{1}, r_{2}\right]$ yields

$$
\begin{aligned}
r_{2}^{n-1} \rho^{\prime}\left(r_{2}\right) & \leq y_{0}+4 \lambda^{2}(-\xi+\varepsilon) \int_{r_{1}}^{r_{2}} \rho(t) t^{n-1} d t \\
& =y_{0 k}+4 \lambda^{2}(-\xi+\varepsilon)\left[\frac{r_{2}^{n}}{n} \rho\left(r_{2}\right)-\frac{r_{1}^{n}}{n} \rho\left(r_{1}\right)-\int_{r_{1}}^{r_{2}} \frac{t^{n}}{n} \rho^{\prime}(t) d t\right] \\
& =y_{0}+4 \lambda^{2}(-\xi+\varepsilon) \frac{r_{2}^{n}}{n} \eta_{1}-4 \lambda^{2}(-\xi+\varepsilon) y_{0} \frac{r_{2}^{2}-r_{1}^{2}}{2 n} \\
& \leq y_{0}\left[1-\frac{4 \lambda^{2}(-\xi+\varepsilon) y_{0}\left(\left(r_{2}^{* 2}-r_{1}^{2}\right) / 2 n\right)}{2 n}\right]+4 \lambda^{2}(-\xi+\varepsilon) \frac{r_{2}^{n}}{n} \eta_{1} \\
& \leq y_{0}^{*}\left[1-\frac{4 \lambda^{2}(-\xi+\varepsilon) y_{0}\left(\left(r *_{2}^{2}-r_{1}^{2}\right) / 2 n\right)}{2 n}\right]+4 \lambda^{2}(-\xi+\varepsilon) \frac{r_{1}^{n}}{n} \eta_{1} .
\end{aligned}
$$

Consequently, in view of (3.58) we obtain $r_{2}^{n-1} \rho^{\prime}\left(r_{2}\right) \leq 0$, a contradiction to (3.45).

Proposition 3.4. For any $\eta_{1} \leq \eta$, there is a positive $\eta_{0} \leq \eta$ such that the solution $\rho=\rho(r)$ with initial value

$$
\rho(0)=-\eta_{0}, \quad \lim _{r \rightarrow 0+} r^{n-1} \rho^{\prime}(r)=0
$$

satisfies

$$
-\eta_{0} \leq \rho(r)<\eta_{1}, \quad r^{n-1} \rho^{\prime}(r) \geq 0, \quad 0<r<r_{2}, \quad r_{2}^{n-1} \rho^{\prime}\left(r_{2}\right)=0
$$

for some $r_{2}>0$.

Proof. By the previous Lemma 3.3, for the given $\eta_{1}$, there exists a $y_{0}^{*}$ such that for all positive $y_{0} \leq y_{0}^{*}$, the solution passing through the point $\left(0, y_{0}\right)$ satisfies inequalities (3.44). On the other hand, in view of Lemma 3.2, there is an $\eta_{0}>0$ such that (3.10) is fulfilled. Therefore, the result follows.

LEMma 3.5. There is a $y_{1}>y_{0}$ such that for any solution $\rho=\rho(r)$ with

$$
\rho\left(r_{1}\right)=0, \quad r_{1}^{n-1} \rho^{\prime}\left(r_{1}\right)=y_{1}
$$

for some $r_{1}>0$, there exist $0<r_{0}<r_{1}<r_{2}$ so that

$$
\begin{array}{clll}
-1 \leq \rho(r)<0, & r^{n-1} \rho^{\prime}(r)>0, & r_{0} \leq r \leq r_{1}, & \rho\left(r_{0}\right)=-1, \\
0 \leq \rho(r)<\xi, & r^{n-1} \rho^{\prime}(r)>0, & r_{1} \leq r \leq r_{2}, & \rho\left(r_{2}\right)=\xi .
\end{array}
$$

Moreover, this solution is a (both sides) nonbounded strictly increasing solution, that is,

$$
\lim _{r \rightarrow 0+} \rho(r)=-\infty, \quad r^{n-1} \rho^{\prime}(r)>0, \quad r \in(0,+\infty), \quad \lim _{r \rightarrow+\infty} \rho(r)=+\infty .
$$

Proof. Supposing first that $n>2$ and that the first conclusion is false. Then for any $y_{1}>y_{0}$,

$$
-1<\rho(r) \leq 0 \quad \forall r \in\left(0, r_{1}\right) .
$$


Now we fix any positive $r_{0}<r_{1}$. By its definition, the nonlinearity $f(\rho),-1 \leq \rho \leq \xi$ is a bounded function, namely,

$$
-4 \lambda^{2} \leq f(\rho) \leq 4 \lambda^{2}, \quad-1 \leq \rho \leq \xi .
$$

So it follows that

$$
\left(r^{n-1} \rho^{\prime}(r)\right)^{\prime} \leq 4 \lambda^{2} r^{n-1}, \quad r_{0} \leq r \leq r_{1},
$$

which in turn implies

$$
r_{1}^{n-1} \rho^{\prime}\left(r_{1}\right)-r^{n-1} \rho^{\prime}(r) \leq 4 \lambda^{2} \frac{r_{1}^{n}-r^{n}}{n}, \quad r_{0} \leq r \leq r_{1},
$$

Consequently, as in the precedent argument, we obtain

$$
\rho\left(r_{0}\right) \leq-m_{1} y_{1}+\frac{4 \lambda^{2}}{n} \frac{r_{1}^{n}}{2-n}\left[\frac{1}{r_{1}^{n-2}}-\frac{1}{r_{0}^{n-2}}\right]-\frac{4 \lambda^{2}}{n}\left[\frac{r_{1}^{2}-r_{0}^{2}}{2}\right],
$$

where

$$
m_{1}=\frac{1}{n-2}\left[\frac{1}{r_{0}^{n-2}}-\frac{1}{r_{1}^{n-2}}\right]>0 \text {. }
$$

Thus, by choosing $y_{1}$ large enough, we conclude the contradiction

$$
\rho\left(r_{0}\right) \leq-1
$$

Similarly, let us assume that for every $y_{1}>0$ and an (also fixed) $r_{2}>r_{1}$, it holds

$$
0 \leq \rho(r)<\xi, \quad r^{n-1} \rho^{\prime}(r)>0, \quad r_{1} \leq r \leq r_{2}, \quad r_{2}^{n-1} \rho^{\prime}\left(r_{2}\right)=0 .
$$

Also by 3.68, we have

$$
\left(r^{n-1} \rho^{\prime}(r)\right)^{\prime} \geq-4 \lambda^{2} r^{n-1}, \quad r_{1} \leq r \leq r_{2},
$$

which implies

$$
r^{n-1} \rho^{\prime}(r)-r_{1}^{n-1} \rho^{\prime}\left(r_{1}\right) \geq-4 \lambda^{2} \frac{r^{n}-r_{1}^{n}}{n}, \quad r_{1} \leq r \leq r_{2}
$$

Hence, as above we obtain (recall that $n>2$ )

$$
\rho\left(r_{2}\right)-\rho\left(r_{1}\right) \geq-\frac{4 \lambda^{2}}{n}\left[\frac{r_{2}^{2}-r_{1}^{2}}{2}-\frac{r_{1}^{n}}{2-n}\left(\frac{1}{r_{2}^{n-2}}-\frac{1}{r_{1}^{n-2}}\right)\right]+\frac{y_{1}}{2-n}\left[\frac{1}{r_{2}^{n-2}}-\frac{1}{r_{1}^{n-2}}\right],
$$

that is, for $y_{1}$ large enough, $\rho\left(r_{2}\right) \geq \xi$, another contradiction. Noticing now the nature of the vector field, we conclude immediately that the obtained solution is a strictly increasing map. 
In order to demonstrate (3.66), we assume that there exists $M>0$ such that for every $y_{1}>y_{0}$,

$$
-M<\rho(r)<M, \quad \forall r \in(0,+\infty)
$$

We suppose first that for any $y_{1}>y_{0}$,

$$
-M<\rho(r) \leq 0, \quad \forall r \in\left(0, r_{1}\right)
$$

and fix any positive $r_{0}<r_{1}$. By its definition, the nonlinearity $f(\rho),-M \leq \rho \leq M$ is a bounded function, namely,

$$
-K \leq f(\rho) \leq K, \quad-M \leq \rho \leq M .
$$

So it follows that

$$
\left(r^{n-1} \rho^{\prime}(r)\right)^{\prime} \leq K r^{n-1}, \quad r_{0} \leq r \leq r_{1}
$$

which in turn implies

$$
r_{1}^{n-1} \rho^{\prime}\left(r_{1}\right)-r^{n-1} \rho^{\prime}(r) \leq K \frac{r_{1}^{n}-r^{n}}{n}, \quad r_{0} \leq r \leq r_{1},
$$

Consequently, as in the preceding argument, we obtain

$$
\rho\left(r_{0}\right) \leq-m_{1} y_{1}+\frac{K}{n} \frac{r_{1}^{n}}{2-n}\left[\frac{1}{r_{1}^{n-2}}-\frac{1}{r_{0}^{n-2}}\right]-\frac{K}{n}\left[\frac{r_{1}^{2}-r_{0}^{2}}{2}\right] .
$$

where

$$
m_{1}=\frac{1}{n-2}\left[\frac{1}{r_{0}^{n-2}}-\frac{1}{r_{1}^{n-2}}\right]>0
$$

Thus, by choosing $y_{1}$ large enough, we conclude the contradiction

$$
\rho\left(r_{0}\right) \leq-M
$$

Similarly, let us assume that for every $y_{1}>0$ and an (also fixed) $r_{2}>r_{1}$, it holds

$$
0 \leq \rho(r)<M, \quad r^{n-1} \rho^{\prime}(r)>0, \quad r_{1} \leq r \leq r_{2}, \quad r_{2}^{n-1} \rho^{\prime}\left(r_{2}\right)=0 .
$$

Also by (3.80), we have

$$
\left(r^{n-1} \rho^{\prime}(r)\right)^{\prime} \geq-K r^{n-1}, \quad r_{1} \leq r \leq r_{2},
$$

which implies

$$
r^{n-1} \rho^{\prime}(r)-r_{1}^{n-1} \rho^{\prime}\left(r_{1}\right) \geq-K \frac{r^{n}-r_{1}^{n}}{n}, \quad r_{1} \leq r \leq r_{2}
$$


Hence, as above we obtain (recall that $n>2$ )

$$
\rho\left(r_{2}\right)-\rho\left(r_{1}\right) \geq-\frac{K}{n}\left[\frac{r_{2}^{2}-r_{1}^{2}}{2}-\frac{r_{1}^{n}}{2-n}\left(\frac{1}{r_{2}^{n-2}}-\frac{1}{r_{1}^{n-2}}\right)\right]+\frac{y_{1}}{2-n}\left[\frac{1}{r_{2}^{n-2}}-\frac{1}{r_{1}^{n-2}}\right],
$$

that is, for $y_{1}$ large enough, $\rho\left(r_{2}\right) \geq M$, another contradiction.

A similar argument works for the case $n=2$ and this clearly ends the proof.

Remark 3.6. We notice that, since the inequality $f(\rho)=(\rho+1) \rho(\rho-\xi)<0$ holds true for $\rho<-1$, the map $r^{n-1} \rho^{\prime}(r)>0,0<r<r_{0}$, is decreasing (see the nature of vector field), hence by the extendability of solutions, $\lim _{r \rightarrow 0+} r^{n-1} \rho^{\prime}(r)=+\infty$ and so $\lim _{r \rightarrow 0+} \rho(r)=$ $-\infty$. Similarly $f(\rho)>0$, for $\rho>\xi$ and this yields $\lim _{r \rightarrow+\infty} \rho(r)=+\infty$.

Remark 3.7. Consider the solution $\rho=\rho(r)$ of the initial value problem (3.3), with (fixed) $-\eta \in(-1,0)$ and let $r_{1}, r_{2}$ be two points such that

$$
\begin{gathered}
-\eta \leq \rho(r)<0, \quad r^{n-1} \rho^{\prime}(r) \geq 0, \quad 0 \leq r<r_{1}, \quad \rho\left(r_{1}\right)=0, \\
\rho(r) \geq 0, \quad r^{n-1} \rho^{\prime}(r) \geq 0, \quad r_{1} \leq r<r_{2} .
\end{gathered}
$$

Since the graph of the function $\lim _{\xi \rightarrow 1} f(\rho)=4 \lambda^{2}\left(\rho^{2}-1\right) \rho$ is symmetric with respect to the $r^{n-1} \rho^{\prime}$-axis, it is clear that

$$
\rho(r)<\xi, \quad r_{1} \leq r<r_{2}
$$

for the case when $\xi$ is close enough to 1 .

Indeed, considering the initial value problem

$$
\begin{gathered}
\left(r^{n-1} \rho^{\prime}(r)\right)^{\prime}=4 \lambda^{2}\left(\rho^{2}-1\right) \rho r^{n-1}, \\
\rho(0)=-\eta, \quad \lim _{r \rightarrow 0+} r^{n-1} \rho^{\prime}(r)=0,
\end{gathered}
$$

if we prove that $(\rho=\rho(r)$ denotes now the solution of IVP (3.92))

$$
\rho(r)<\eta, \quad r \geq 0
$$

by the continuity of solutions upon the nonlinearity, at the case when $\xi \rightarrow 1-$, the boundary value problem (3.1) does not admit any solution.

Suppose in the contrary, that there exists a point $\hat{r}_{2}>\widehat{r}_{1}=r_{1}$ such that

$$
\rho\left(\hat{r}_{2}\right)=\eta, \quad 0<m_{0}=\hat{r}_{2}^{n-1} \rho^{\prime}\left(\hat{r}_{2}\right) \leq \hat{r}_{1}^{n-1} \rho^{\prime}\left(\hat{r}_{1}\right)=m_{1} .
$$

Then there exist a point $\hat{r}_{0} \in\left(0, \hat{r}_{1}\right)$ such that $\hat{r}_{0}^{n-1} \rho^{\prime}\left(\hat{r}_{0}\right)=m_{1}$ and furthermore, for any $t \in\left(\hat{r}_{1}, \hat{r}_{2}\right)$, there is an $r \in\left(\hat{r}_{0}, \hat{r}_{1}\right)$ with

$$
r^{n-1} \rho^{\prime}(r)=t^{n-1} \rho^{\prime}(t) .
$$


Since $r<t$, it follows that (for all such $r$ and $t$ )

$$
\rho^{\prime}(r)>\rho^{\prime}(t)
$$

Consider now a partition $\left\{m_{0}<m_{1}<\cdots<m_{k}\right\}$ of the interval $\left[m_{0}, m_{1}\right]$ as well as the corresponding partitions

$$
\left\{\hat{r}_{0}=r_{0}<r_{1}<\cdots<r_{k}=\hat{r}_{1}\right\}, \quad\left\{\hat{r}_{2}=t_{0}>t_{1}>\cdots>t_{k}=\hat{r}_{1}\right\}
$$

of $\left[\hat{r}_{0}, \hat{r}_{1}\right]$ and $\left[\hat{r}_{1}, \hat{r}_{2}\right]$, respectively, so that

$$
r_{i}^{n-1} \rho^{\prime}\left(r_{i}\right)=t_{i}^{n-1} \rho^{\prime}\left(t_{i}\right), \quad(i=0,1, \ldots, k) .
$$

Then, of course,

$$
\rho^{\prime}\left(r_{i}\right)>\rho^{\prime}\left(t_{i}\right), \quad(i=0,1, \ldots, k)
$$

In addition, because the map $\rho^{\prime}=\rho^{\prime}(t), \hat{r}_{0} \leq r \leq \widehat{r}_{2}$, is continuous (and bounded), we can choose the $\max \left\{m_{i}-m_{i-1}: i=1,2, \ldots, k\right\}$ small enough, so that

$$
\frac{t_{i+1}-t_{i}}{2} \rho^{\prime}\left(t_{i}\right) \leq \frac{r_{i+1}-r_{i}}{2} \rho^{\prime}\left(r_{i}\right), \quad(i=0,1, \ldots, k-1) .
$$

Hence

$$
\sum_{i=1}^{k} \frac{t_{i+1}-t_{i}}{2} \rho^{\prime}\left(t_{i}\right) \leq \sum_{i=1}^{k} \frac{r_{i+1}-r_{i}}{2} \rho^{\prime}\left(r_{i}\right)
$$

and thus we obtain the contradiction

$$
\eta=\rho\left(\hat{r}_{2}\right)=\int_{\widehat{r}_{1}}^{\hat{r}_{2}} \rho^{\prime}(t) d t \leq \int_{\hat{r}_{0}}^{\hat{r}_{1}} \rho^{\prime}(r) d r<\int_{0}^{\hat{r}_{1}} \rho^{\prime}(r) d r=-\rho(0)=\eta .
$$

In conclusion, (3.93) and so (3.91) hold true. In others words, using the terminology of the previous section, for all large enough $\xi \in(0,1)$, we have

$$
\mathscr{K}\left(P_{0}\right) \subset E^{*}:=\left\{\left(\rho, p \rho^{\prime}\right) \in \partial \omega: p \rho^{\prime}=0\right\}, \quad P_{0}=(-\eta, 0) .
$$

On the other hand, when $\xi \rightarrow 0+$, there always exists a solution $\rho=\rho(r)$ of the IVP (3.3) such that

$$
\begin{aligned}
& -\eta \leq \rho(r)<0, \quad r^{n-1} \rho^{\prime}(r) \geq 0, \quad 0 \leq r<r_{1}, \quad \rho\left(r_{1}\right)=0, \\
& 0 \leq \rho(r)<\xi, \quad r^{n-1} \rho^{\prime}(r)>0, \quad r_{1} \leq r<r_{2}, \quad \rho\left(r_{2}\right)=\xi \text {, }
\end{aligned}
$$

that is,

$$
\mathscr{K}\left(P_{0}\right) \subset E_{1}^{*}=\left\{\left(\rho, p \rho^{\prime}\right) \in \partial \omega: \rho=\xi\right\} .
$$

Theorem 3.8. For every small enough $\xi \in(0,1)$, the boundary value problem (3.1) admits (at least) one strictly increasing solution. 
Proof. In view of Proposition 3.4, for a given $\xi$, there is an $\eta_{1}^{*}>0$ small enough and a solution $\rho=\rho_{0}(r)$ of the IVP (3.3), such that (3.103) is satisfied, with $P_{0}=\left(-\eta_{1}^{*}, 0\right)$. On the other hand, since $\xi$ is small, there exists an $\eta_{0}^{*} \in(0,1)$ large enough and a solution $\rho=\rho_{1}(r)$ with

$$
\begin{gathered}
-\eta_{0}^{*} \leq \rho(r)<0, \quad r^{n-1} \rho^{\prime}(r) \geq 0, \quad 0 \leq r<r_{1}, \quad \rho(0)=-\eta_{0}^{*}, \quad \rho\left(r_{1}\right)=0, \\
0 \leq \rho(r)<\xi, \quad r^{n-1} \rho^{\prime}(r)>0, \quad r_{1} \leq r<r_{2}, \quad \rho\left(r_{2}\right)=\xi
\end{gathered}
$$

for some positive values $r_{1}$ and $r_{2}$ of the variable $r$, that is, (3.105) is also fulfilled with $P_{0}=\left(-\eta_{0}^{*}, 0\right)$.

Considering finally the continuum set

$$
E_{0}:=\left[-\eta_{0}^{*},-\eta_{1}^{*}\right] \times\{0\}
$$

we may apply Theorem 2.9 to get an $\eta \in\left[-\eta_{0}^{*},-\eta_{1}^{*}\right]$ and the unique solution $\rho=\rho(r) \in$ $\mathscr{L}(P), P=(\eta, 0)$ of the initial value problem (3.3) such, that $\lim _{r \rightarrow+\infty} \rho(r)=\xi$.

Conjecture 3.9. If we know that the above obtained singular point $P=(\eta, 0)$ is unique, then by Theorem 2.9, the corresponding solution $\rho \in \mathscr{X}(P)$ is also unique. Numerical trials indicate that is true! However this actually is an open problem.

Remark 3.10. The above obtained solution of the boundary value problem (3.1), transferring via the transformation given above of (1.8), clearly gives a positive solution $\rho=\rho(r)$ of our problem (1.5)-(1.7), that is,

$$
0<\rho_{1}<\rho(r)<\rho_{l}, \quad 0<r<+\infty .
$$

Theorem 3.11. A unique $\xi_{M} \in(0,1)$ exists such that the terminal value problem

$$
\begin{gathered}
\frac{1}{r^{n-1}}\left(r^{n-1} \rho^{\prime}(r)\right)^{\prime}=4 \lambda^{2}(\rho+1) \rho\left(\rho-\xi_{M}\right):=f(\rho), \\
\lim _{r \rightarrow 0+} \rho(r)=-1, \quad \lim _{r \rightarrow+\infty} \rho(r)=\xi_{M}
\end{gathered}
$$

admits at least one strictly increasing solution.

Furthermore, the point $\xi_{M} \in(0,1)$ is the maximal one in the sense that, for every $\xi>\xi_{M}$, the boundary value problem (3.1) does not admit any solution.

Proof. We consider a fixed $\xi \in(0,1)$ and notice Lemma 3.2. Then for any (small) $y_{0}>0$ there exists an $\eta_{0} \in[0,1)$ and $r_{1}>0$ such that the solution of IVP

$$
\begin{gathered}
\frac{1}{r^{n-1}}\left(r^{n-1} \rho^{\prime}(r)\right)^{\prime}=4 \lambda^{2}(\rho+1) \rho(\rho-\xi), \\
\rho(0)=-\eta_{0}, \quad \lim _{r \rightarrow 0+} r^{n-1} \rho^{\prime}(r)=0
\end{gathered}
$$


satisfies

$$
-\eta_{0} \leq \rho(r)<0, \quad \rho\left(r_{1}\right)=0, \quad 0 \leq r^{n-1} \rho^{\prime}(r) \leq y_{0}, \quad 0 \leq r<r_{1} .
$$

In view of Lemma 3.5, there is a $y_{1}>y_{0}$ such that the solution $\rho=\rho(r)$ which satisfies $\rho\left(r_{1}\right)=0$ and $r_{1}^{n-1} \rho^{\prime}\left(r_{1}\right)=y_{1}$, for some $r_{1}>0$, there exists an $r_{0} \in\left(0, r_{1}\right)$ such that

$$
-1 \leq \rho(r)<0, \quad r^{n-1} \rho^{\prime}(r)>0, \quad r_{0} \leq r \leq r_{1}, \quad \rho\left(r_{0}\right)=-1 .
$$

Consider the continuum

$$
E_{0}=\{0\} \times\left[y_{0}, y_{1}\right]
$$

in the domain

$$
\Omega:=\left\{\left(\rho, p \rho^{\prime}\right):-1 \leq \rho \leq 0, r^{n-1} \rho^{\prime} \geq 0\right\}
$$

By the sign property of the nonlinearity (nature of the vector field), it is clear that every solution $\rho \in \mathscr{L}\left(E_{0}\right)$ extended backwards is a strictly increasing function. Therefore, by the fundamental continuation theorem, we can define a map

$$
\mathscr{K}^{*}: E_{0} \longrightarrow 2^{\partial \Omega}
$$

analogously with the similarly defined one above, by

$$
\mathscr{K}^{*}(P):=\left\{\left(\rho\left(r_{0}\right), r_{0}^{n-1} \rho^{\prime}\left(r_{0}\right)\right) \in \partial \Omega: \rho \in \mathscr{X}(P), P=(0, y) \in E_{0}\right\}
$$

for some $r_{0} \in\left(0, r_{1}\right)$. Consider the subsets

$$
E_{-1}^{*}=\left\{\left(\rho, r^{n-1} \rho^{\prime}\right) \in \partial \Omega: \rho=-1\right\}, \quad E_{*}:=\left\{\left(\rho, r^{n-1} \rho^{\prime}\right) \in \partial \Omega: r^{n-1} \rho^{\prime}=0\right\}
$$

of $\Omega$, and notice that both sets

$$
\mathscr{K}^{*}\left(E_{0}\right) \cap E_{-1}^{*}, \quad \mathscr{K}^{*}\left(E_{0}\right) \cap E_{*}
$$

are nonempty connected subsets of the boundary $\partial \Omega$. Consequently, in view of Lemma 2.6, we must have

$$
\partial\left(\mathscr{K}^{*}\left(E_{0}\right) \cap E_{*}\right) \cap E_{-1}^{*}=\{(-1,0)\} \neq \varnothing .
$$

This means that there exists a singular point $P \in E_{0}$ of the map $\mathscr{K}^{*}$, that is, there is a solution $\rho=\rho(r) \in \mathscr{X}(P)$ which remains left asymptotic in $\Omega$ and so it satisfies the left asymptotic relations in (3.109). 
Finally, noticing Remark 3.7, for the case where $\xi=\xi_{1}$ is close enough to the right end of the interval $(0,1)$, there is an $r_{2}>0$ such that

$$
0 \leq \rho_{1}(r)<\xi_{1}, \quad r^{n-1} \rho_{1}^{\prime}(r) \geq 0, \quad r_{1} \leq r \leq r_{2}, \quad r_{2}^{n-1} \rho_{1}^{\prime}\left(r_{2}\right)=0,
$$

and mainly $\rho_{1}\left(r_{2}\right)<\xi_{1}$, where $\rho=\rho_{1}(r)$ is a solution of the equation in (3.109), with $\xi_{M}$ replaced by $\xi_{1}$ such that $\lim _{r \rightarrow 0+} \rho_{1}(r)=-1$.

On the other hand, there exists a $\xi_{0}<\xi_{1}$ such that (now $\rho=\rho_{0}(r)$ is a solution of the equation in (3.109), with $\xi_{M}$ replaced by $\xi_{0}$ and the new $r_{2}$ generally different by the above one)

$$
\lim _{r \rightarrow 0+} \rho_{0}(r)=-1, \quad \rho_{0}\left(r_{2}\right)=\xi_{0}, \quad r^{n-1} \rho_{0}^{\prime}(r)>0, \quad 0<r \leq r_{2}
$$

for (at least) one such solution. This is obvious, since for $\xi=0$, the nonlinearity $f(\rho)=$ $4 \lambda^{2}(\rho+1) \rho^{2}>0, \rho \geq-1$, that is, the function $r^{n-1} \rho^{\prime}(r)$ is strictly increasing and thus $\lim _{r \rightarrow+\infty} \rho(r)=+\infty$.

Now we set $\xi_{0}=\xi_{00}, \xi_{1}=\xi_{10}$, and

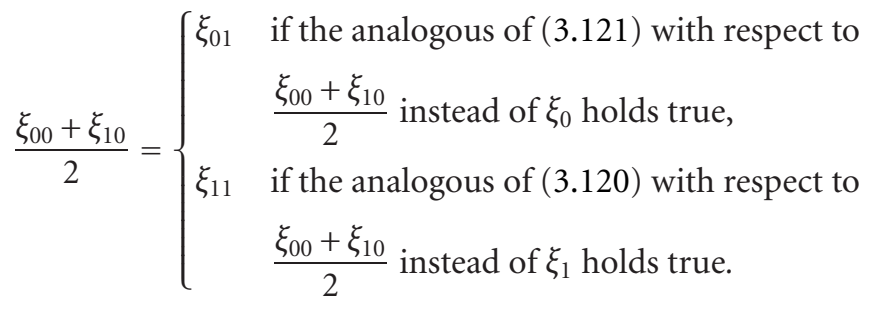

This definition of $\xi_{01}$ and $\xi_{11}$ is well posed because, since the function $r^{n-1} \rho^{\prime}(r)$ is decreasing on $\left[r_{1}, r_{2}\right]$ and $\rho=\rho(r)$ is an increasing one on $\left[r_{1}, r_{2}\right]$, we may apply the usual continuation theorem to guarantee that there is not other case. We repeat this procedure replacing the interval $\left[\xi_{01}, \xi_{10}\right]$ or $\left[\xi_{00}, \xi_{11}\right]$, according to $(3.121)$ or $(3.120)$, with $\left[\xi_{01}, \xi_{11}\right]$ to get a second interval $\left[\xi_{02}, \xi_{12}\right]$ with same as $\left[\xi_{01}, \xi_{11}\right]$ properties and so forth and finally we can obtain sequences $\left\{\xi_{0 n}\right\}$ and $\left\{\xi_{1 n}\right\}$ such that

$$
\lim \xi_{0 n}=\lim \xi_{1 n}=\xi_{M}
$$

By the construction of $\left\{\xi_{\text {in }}\right\}(i=0,1)$ and the definition of $\xi_{M}$, we conclude that the BVP (3.109) is solvable.

The last result for the maximality of $\xi_{M} \in(0,1)$ follows by the monotonicity of $\left\{\xi_{\text {in }}\right\}$.

Remark 3.12. If the singular point $P_{0}$ of the map $\mathscr{K}^{*}$ is unique, then the uniqueness of the point $\xi_{M}$ and the uniqueness of solutions with respect to their initial data function $\rho_{M}(r)$ yield the uniqueness of the above obtained solution $\rho=\rho_{M}(r), 0<r<+\infty$. This remains also an open problem. Some monotonicity assumptions on the nonlinearity, possibly, are sufficient for that. 


\section{A numerical approach}

By the previous and especially in view of Theorems 3.8 and 3.11, it is obvious that we cannot find out theoretically the maximal point $\xi_{M}$ and (then an initial one) $\rho_{0}$ such that the BVP (3.1) admits an increasing solution. But if we know that for some $\xi^{*}$ there is an initial point $\rho_{0}^{*}$ such that the corresponding solution $\rho=\rho(r)$ satisfies for some $r_{1}>0$,

$$
\rho(0)=\rho_{0}^{*}, \quad \rho_{0}^{*}<\rho(r)<\xi^{*}, \quad r^{n-1} \rho^{\prime}(r)>0, \quad 0<r<r_{1}, \quad \rho\left(r_{1}\right)=\xi^{*},
$$

then we can approximate numerically the solution of (3.1), for every $\xi \in\left(0, \xi^{*}\right]$, using the NDSolve command of MATHEMATICA and applying the shooting method. So, we restrict our consideration in the sequel for the case $n=3$ and $\lambda=1$. Precisely, by the series expression (3.4)-(3.5) of the solutions, we may use as initial values

$$
\rho\left(r_{0}\right)=\rho_{0}, \quad r_{0}^{n-1} \rho^{\prime}\left(r_{0}\right)=(4 / 3) r_{0}^{n} \lambda^{2}\left(\rho_{0}+1\right) \rho_{0}\left(\rho_{0}-\xi\right)
$$

for a small enough $r_{0}$. In this way for $r_{0}=0.01$, and $\xi=0.6616, \rho_{0}=-0.999112$ or $\xi=0.6617, \rho_{0}=-0.999112$, we obtained the two curves on the phase plane $\left(\rho, r^{n-1} \rho^{\prime}\right)$, respectively, (see Figures 2.1 and 2.2). We notice that at the first case the relations

$$
\rho(0)=\rho_{0}, \quad \rho_{0}<\rho(r)<\xi, \quad r^{n-1} \rho^{\prime}(r)>0, \quad 0<r<r_{1}, \quad \rho\left(r_{1}\right)=\xi
$$

are fulfilled, while at the second one, we have

$$
\rho(0)=\rho_{0}, \quad \rho_{0} \leq \rho(r)<\xi, \quad r^{n-1} \rho^{\prime}(r) \geq 0, \quad 0 \leq r \leq r_{1}, \quad r_{1}^{n-1} \rho^{\prime}\left(r_{1}\right)=0 .
$$

Following the same technique, we get the next two Figures 2.3 and 2.4 and notice that in view of the last one, it seems that $\xi=0.83428 \simeq \xi_{M}$ is a "good" approximation of the extreme (existence) point $\xi_{M}$ according to Theorem 3.11.

We notice finally, for the convenience of the reader, that we have used the next NDSolve command of MATHEMATICA:

$$
\xi=0.8 ; \quad \rho_{0}=-0.9999997 ; \quad r_{0}=0.01 ; \quad \rho_{0}^{\prime}=(4 / 3)\left(\rho_{0}+1\right) \rho_{0}\left(\rho_{0}-\xi\right) r_{0}^{3} ;
$$

solution

$$
\begin{aligned}
=\text { NDSolve }[ & \left\{p_{1}^{\prime}[r]=p_{2}[r], r^{2} p_{2}^{\prime}[r]+2 r p_{2}[r]=4 r^{2}\left(p_{1}[r]+1\right) p_{1}[r]\left(p_{1}[r]-\xi\right),\right. \\
& \left.\left.p_{1}\left[r_{0}\right]=\rho_{0}, r_{0}^{2} p_{2}\left[r_{0}\right]=\rho_{0}\right\},\left\{p_{1}[r], p_{2}[r]\right\},\{r, 0.1,12\}, \text { MaxSteps- }>10^{3}\right] .
\end{aligned}
$$

ParametricPlot [Evaluate $\left[\left\{p_{1}[r], r^{2} \backslash p_{2}[r]\right\} /\right.$. solution],$\left.\{r, 0,12\}\right]$.

Remark 4.1. Following the same technique, we may prove the following existence result (see Figure 4.1). 


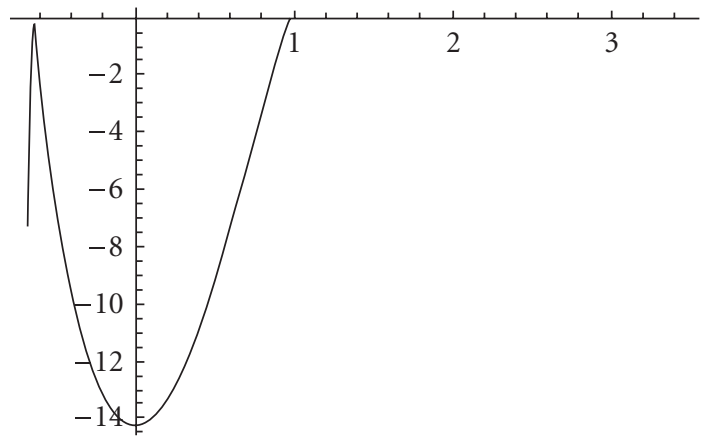

Figure 4.1. $\left(\rho_{0} \simeq 0.999112, \xi \simeq-0.6372\right)$.

There is a minimum $\xi_{m} \in(-1,0)$ such that for every $\xi \in\left(\xi_{m}, 0\right)$, there exists a $\rho_{0} \in$ $(0,1)$ such that the solution $\rho \in \mathscr{L}\left(P_{0}\right), P_{0}=\left(\rho_{0}, 0\right)$ of the IVP

$$
\begin{gathered}
\frac{1}{r^{n-1}}\left(r^{n-1} \rho^{\prime}(r)\right)^{\prime}=4 \lambda^{2}(\rho-1) \rho(\rho-\xi), \\
\lim _{r \rightarrow 0+} r^{n-1} \rho^{\prime}(r)=0, \quad \rho(0)=\rho_{0}
\end{gathered}
$$

is a strictly decreasing function, which satisfies

$$
\lim _{r \rightarrow+\infty} r^{n-1} \rho^{\prime}(r)=0, \quad \lim _{r \rightarrow+\infty} \rho(r)=\xi
$$

Furthermore, there exists a strictly decreasing solution $\rho_{m}$ of the terminal value problem

$$
\begin{gathered}
\frac{1}{r^{n-1}}\left(r^{n-1} \rho^{\prime}(r)\right)^{\prime}=4 \lambda^{2}(\rho-1) \rho\left(\rho-\xi_{m}\right), \\
\lim _{r \rightarrow 0+} \rho(r)=1, \quad \lim _{r \rightarrow+\infty} \rho(r)=\xi_{m} .
\end{gathered}
$$

Also, there is a (monoparametric) family of strictly decreasing solutions

$$
\lim _{r \rightarrow 0+} \rho(r)=+\infty, \quad r^{n-1} \rho^{\prime}(r)<0, \quad-\infty<r<+\infty, \quad \lim _{r \rightarrow+\infty} \rho(r)=-\infty .
$$

Finally by Proposition 3.4 and taking into account Remark 3.7, we presume (Figure 4.2). Conjecture 4.2. For any $\xi \in(0,1)$, there is a maximal $\rho_{0 M} \in(0,1)$ such that the solution of IVP

$$
\begin{gathered}
\frac{1}{r^{n-1}}\left(r^{n-1} \rho^{\prime}(r)\right)^{\prime}=4 \lambda^{2}(\rho+1) \rho(\rho-\xi):=f(\rho), \\
\lim _{r \rightarrow 0+} r^{n-1} \rho^{\prime}(r)=0, \quad \lim _{r \rightarrow 0} \rho(r)=\rho_{0},
\end{gathered}
$$

with $0<\rho_{0}<\rho_{0 M}$, is oscillating and asymptotically stable, that is, $\lim _{r \rightarrow+\infty} \rho(r)=0$. 


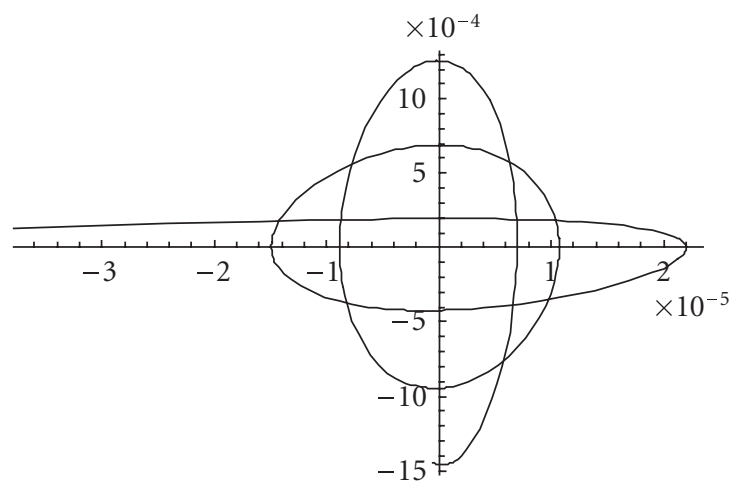

Figure 4.2. $\left(\rho_{0} \simeq-0.0001, \xi \simeq 0.6597253\right)$.

\section{Acknowledgment}

We thank referees for their careful reading and deep understanding of this paper, as well as for their significant comments, which helped us to improve the manuscript considerably.

\section{References}

[1] R. P. Agarwal and I. Kiguradze, Two-point boundary value problems for higher-order linear differential equations with strong singularities, Boundary Value Problems 2006 (2006), Article ID 83910, 32 pages.

[2] J. V. Baxley, Boundary value problems on infinite intervals, Boundary Value Problems for Functional-Differential Equations (J. Henderson, ed.), World Scientific, New Jersey, 1995, pp. 49-62.

[3] H. Berestycki, P.-L. Lions, and L. A. Peletier, An ODE approach to the existence of positive solutions for semilinear problems in $\mathbf{R}^{N}$, Indiana University Mathematics Journal 30 (1981), no. 1, 141157.

[4] D. Bonheure, J. M. Gomes, and L. Sanchez, Positive solutions of a second-order singular ordinary differential equation, Nonlinear Analysis 61 (2005), no. 8, 1383-1399.

[5] W. A. Copel, Stability and Asymptotic Behavior of Differential Equations, Heath, Massachusetts, 1965.

[6] F. Dell'Isola, H. Gouin, and G. Rotoli, Nucleation of spherical shell-like interfaces by second gradient theory: numerical simulations, European Journal of Mechanics. B. Fluids 15 (1996), no. 4, $545-568$.

[7] F. Gazzola, J. Serrin, and M. Tang, Existence of ground states and free boundary problems for quasilinear elliptic operators, Advances in Differential Equations 5 (2000), no. 1-3, 1-30.

[8] K. Kuratowski, Topology II, Academic Press, New York, 1968.

[9] P. K. Palamides, Singular points of the consequent mapping, Annali di Matematica Pura ed Applicata. Serie Quarta 129 (1981), 383-395 (1982).

[10] B__ Boundary-value problems for shallow elastic membrane caps, IMA Journal of Applied Mathematics 67 (2002), no. 3, 281-299.

[11] P. K. Palamides and G. N. Galanis, Positive, unbounded and monotone solutions of the singular second Painlevé equation on the half-line, Nonlinear Analysis 57 (2004), no. 3, 401-419.

[12] Y. Rocard, Thermodynamique, chapter 5, Masson, Paris, 1967. 


\section{A terminal BVP}

[13] W. Walter, Ordinary Differential Equations, Graduate Texts in Mathematics, vol. 182, Springer, New York, 1998.

[14] W. Wasov, Asymptotic Expressions for Ordinary Differential Equations, John Wiley \& Sons, New York, 1965.

Alex P. Palamides: Department of Telecommunications Science and Technology, University of Peloponesse, 22100 Tripolis, Greece

E-mail address: palamid@uop.gr

Theodoros G. Yannopoulos: Department of Mathematics, Technological Educational Institute (TEI) of Athens, 12210 Egaleo, Greece

E-mail address: thyann@teiath.gr 